\title{
Albumin-Consolidated AIEgens for Boosting Glioma and Cerebrovascular NIR-II Fluorescence Imaging
}

\author{
Duyang Gao ${ }^{1+t}$, Yaxi Li ${ }^{2+}$, Yayun $W^{1}{ }^{1}$, Yu Liu ${ }^{1}$, Dehong $H u^{1}$, Simin Liang ${ }^{3}$, Jiuling
}

Liao ${ }^{1}$, Min Pan ${ }^{3}$, Pengfei Zhang ${ }^{1}$, Kai Li $^{2}$, Xin Liul ${ }^{1}$, Hairong Zhengl ${ }^{*}$, Zonghai

Sheng ${ }^{1 *}$

1. Paul C. Lauterbur Research Center for Biomedical Imaging, CAS key laboratory of health informatics, Shenzhen Key Laboratory of Ultrasound Imaging and Therapy, Institute of Biomedical and Health Engineering, Shenzhen Institutes of Advanced Technology, Chinese Academy of Sciences, Shenzhen, 518055, P. R. China. Email: hr.zheng@siat.ac.cn; zh.sheng@siat.ac.cn

2. Department of Biomedical Engineering, SUSTech Academy for Advanced Interdisciplinary Studies, Southern University of Science and Technology (SUSTech), Shenzhen, 518055 China

3. Department of Ultrasonography, Shenzhen Hospital of Guangzhou University of Chinese Medicine, Shenzhen, China, 518034

KEYWORDS: Fluorescence imaging, Second near-infrared window, AIE nanoprobes, Brain diseases, Albumin 

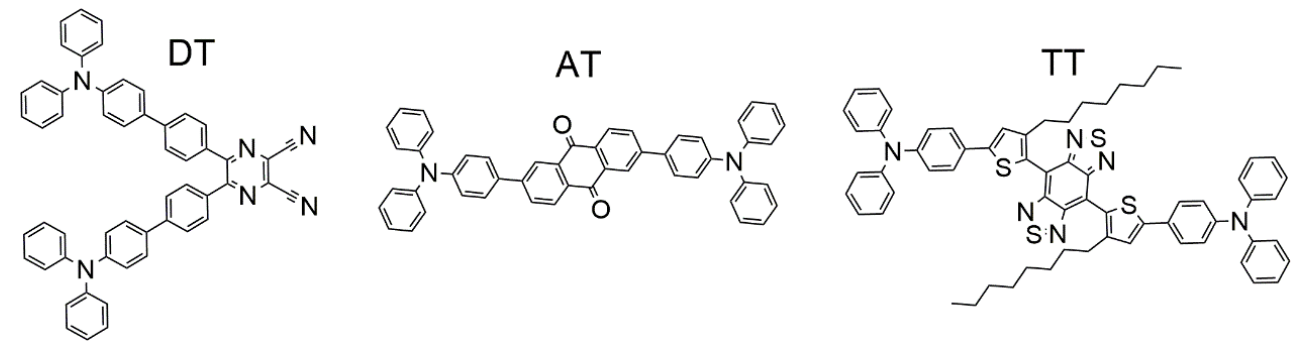

Figure S1. Molecular structures of the AlEgens.

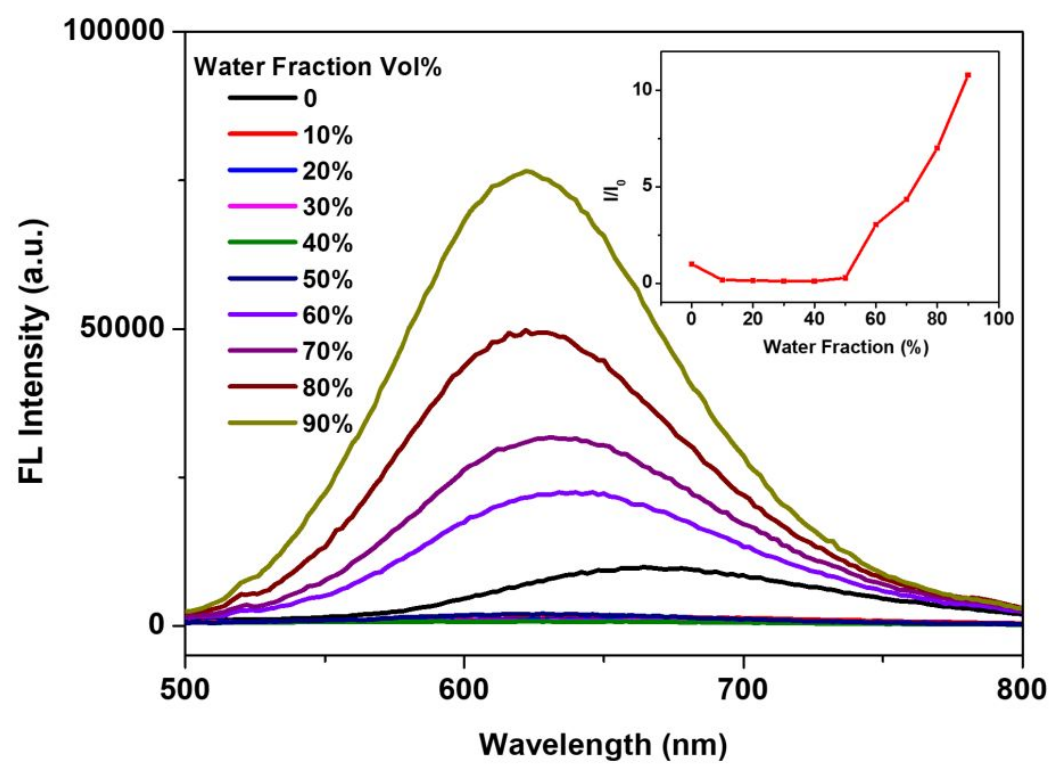

Figure S2. FL spectra of DT AIEgens in THF- $\mathrm{H}_{2} \mathrm{O}$ mixtures with different water fractions (fw). Inset: Plot of the relative FL intensity (I/I0) at $630 \mathrm{~nm}$ versus fw of the THF- $\mathrm{H}_{2} \mathrm{O}$ mixture of DT AIEgens. Excitation wavelength: $440 \mathrm{~nm}$. 


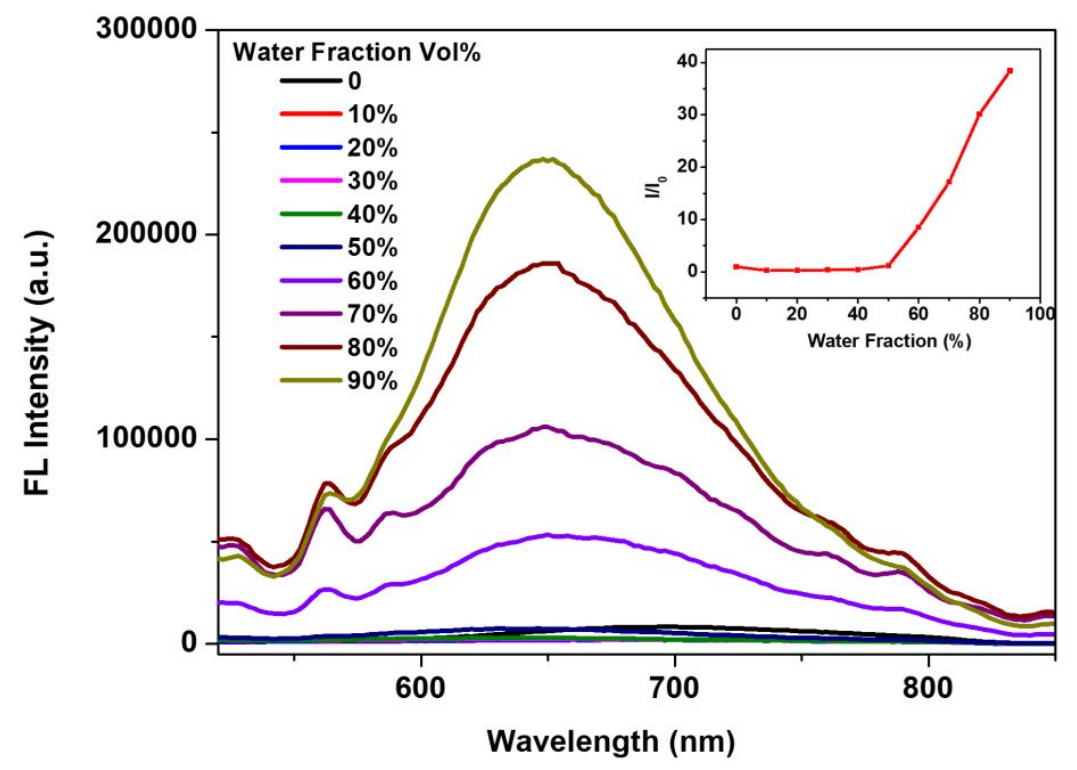

Figure S3. FL spectra of AT AIEgens in THF- $\mathrm{H}_{2} \mathrm{O}$ mixtures with different water fractions (fw). Inset: Plot of the relative FL intensity (I/I0) at $650 \mathrm{~nm}$ versus fw of the THF- $\mathrm{H}_{2} \mathrm{O}$ mixture of AT AIEgens. Excitation wavelength: $470 \mathrm{~nm}$.

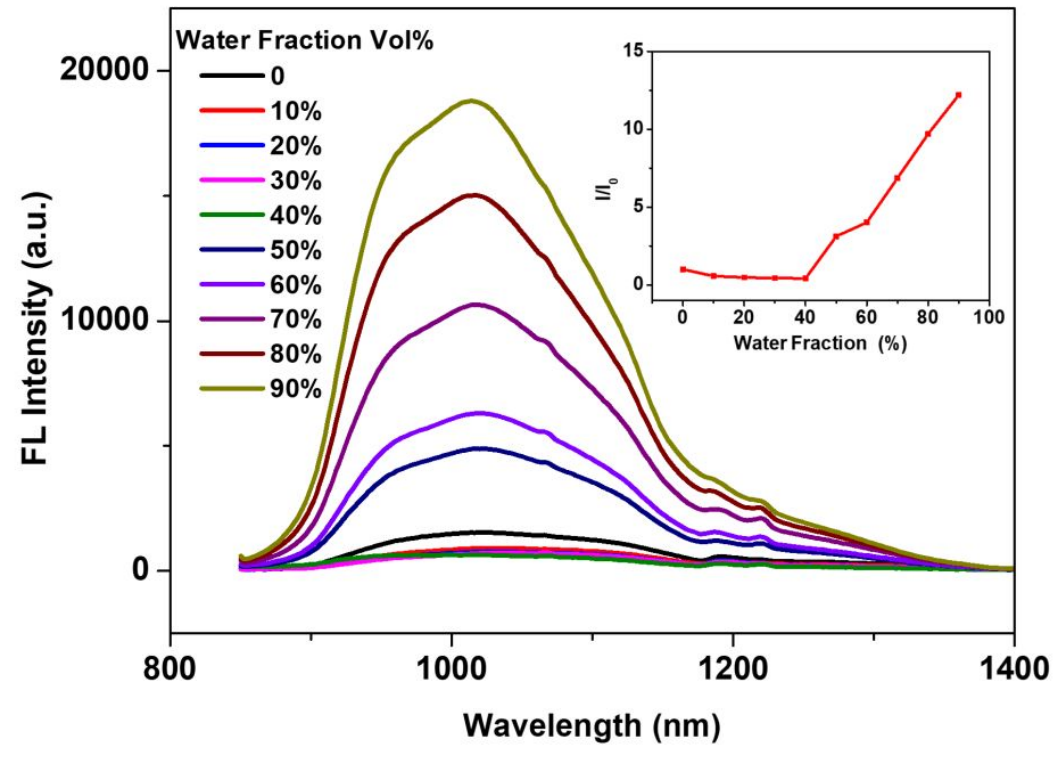

Figure S4. FL spectra of TT AIEgens in THF- $\mathrm{H}_{2} \mathrm{O}$ mixtures with different water fractions (fw). Inset: Plot of the relative FL intensity (I/I0) at $1016 \mathrm{~nm}$ versus fw of the THF- $\mathrm{H}_{2} \mathrm{O}$ mixture of TT AIEgens. Excitation wavelength: $808 \mathrm{~nm}$. 


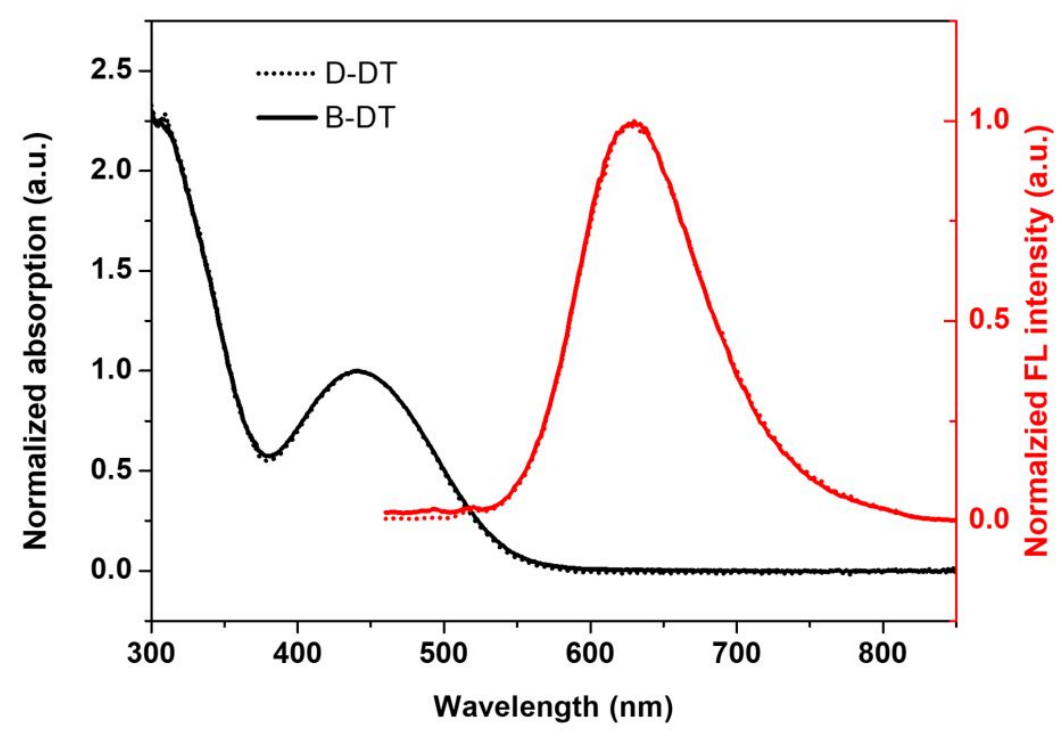

Figure S5. Uv-Vis absorption and FL emission spectra of D-DT and B-DT AIE dots in PBS

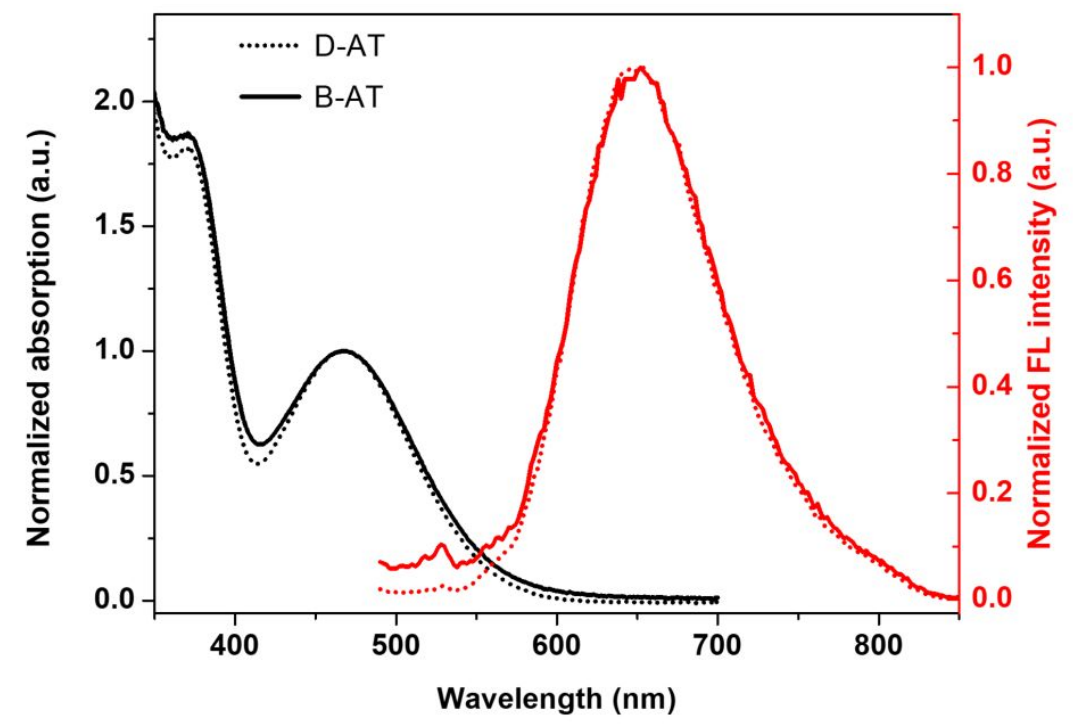

Figure S6. Uv-Vis absorption and FL emission spectra of D-AT and B-AT AIE dots in PBS 


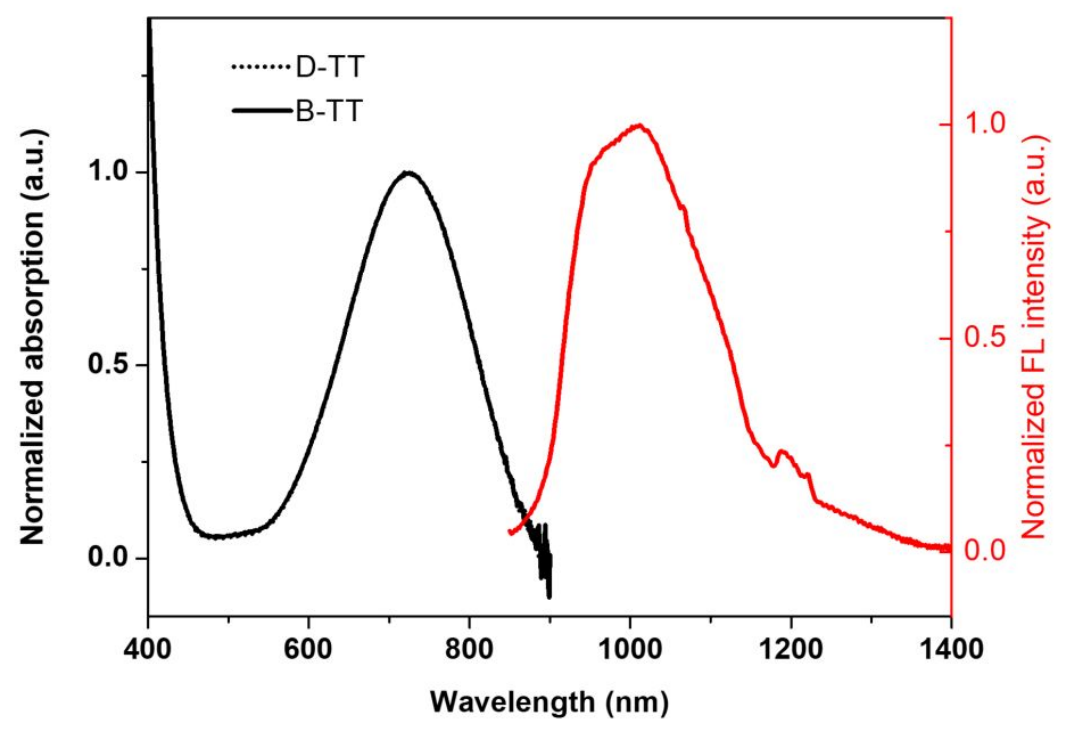

Figure S7. Uv-Vis absorption and FL emission spectra of D-TT and B-TT AIE dots in PBS
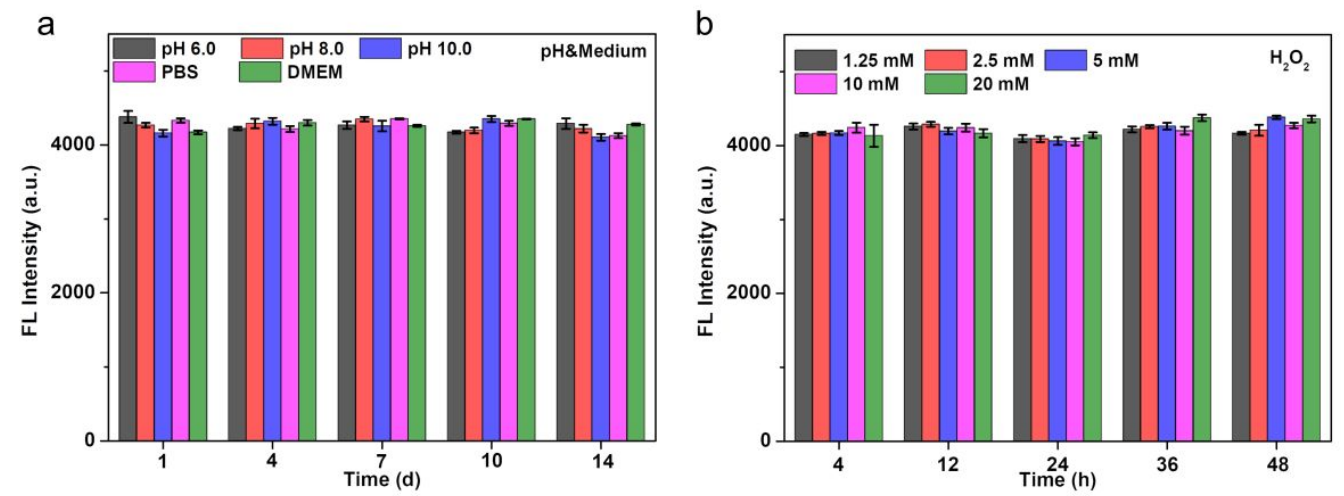

Figure S8. Stability of the B-TT AIE dots. a) FL intensity of B-TT AIE dots in buffer with different $\mathrm{pH}$ values and mediums; b) The FL intensity variation of the B-TT AIE dots in the presence of $\mathrm{H}_{2} \mathrm{O}_{2}$ with different concentrations. 